\title{
Efficacy and safety of bevacizumab-based combination therapy for treatment of patients with metastatic colorectal cancer
}

REVIEW

This article was published in the following Dove Press journal: OncoTargets and Therapy

\author{
$\operatorname{Ran} X u^{1,2, *}$ \\ Chen $\mathrm{Xu}^{1, *}$ \\ Chuntong $\mathrm{Liu}^{3}$ \\ Can Cui ${ }^{4}$ \\ Jing $\mathrm{Zhu}^{5}$
}

'Medical School of Nantong University, Jiangsu 22600 I, China; ${ }^{2}$ Huai'an Key Laboratory of Gastrointestinal Cancer, Jiangsu 22300I, China; ${ }^{3}$ XuZhou Medical University, Jiangsu 221000 , China; ${ }^{4}$ Macau University of Science and Technology, Macau 519020, China; ${ }^{5}$ Department of Oncology, The Affiliated Huaian NO I People's Hospital of Nanjing Medical University, Jiangsu 22300I, China

*These authors contributed equally to this work
Correspondence: Ran $\mathrm{Xu}$

Medical School of Nantong University, 19 Qixiu Road, Jiangsu 22600I, China

Email ranxu1996@I63.com

Jing Zhu

Department of Oncology, The Affiliated Huaian NO I People's Hospital of Nanjing Medical University, 6 Beijing West Road, Jiangsu 22300I, China Email zhujingbiology@।63.com
Aim: The use of bevacizumab in combination therapy is an emerging trend in metastatic colorectal cancer treatment. However, the clinical value of different combination types remains under debate. Thus, a meta-analysis of randomized controlled trials (RCTs) comparing bevacizumab-based combination therapy with monotherapy (therapy that uses one type of treatment, such as chemotherapy or surgery alone, to treat metastatic colorectal cancer) was performed, aiming to evaluate the safety and efficacy of bevacizumab-based combination therapy and to find a more beneficial combination.

Methods: We searched for clinical studies that evaluated bevacizumab-based combination therapy in metastatic colorectal cancer. We extracted data from these studies to evaluate the relative risk (RR) of overall response rate (ORR) and grade $3 / 4$ treatment-related adverse events (AEs), HRs of overall survival (OS), and progression-free survival (PFS).

Results: Eight RCTs were identified $(n=3,424)$. Treatments included combinations of bevacizumab and oxaliplatin, fluorouracil, and leucovorin (FOLFOX4), combinations of bevacizumab and capecitabine and oxaliplatin, combinations of bevacizumab and fluorouracil/leucovorin, combinations of bevacizumab and irinotecan, fluorouracil, and leucovorin (IFL), and combinations of bevacizumab and capecitabine. Bevacizumab-based combination therapy showed higher ORR (RR: $1.40 ; 95 \%$ CI: $1.10-1.78 ; P=0.005$ ), PFS (HR: $0.64 ; 95 \%$ CI: $0.55-0.73 ; P=0.000$ ), and OS (HR: $0.82 ; 95 \%$ CI: $0.73-0.92 ; P=0.001$ ) values than monotherapy. However, higher grade $3 / 4$ treatment-related AEs (RR: $1.27 ; 95 \%$ CI: $1.15-1.41 ; P=0.000$ ) were observed in combination therapy than in monotherapy.

Conclusion: This meta-analysis showed that the addition of IFL to bevacizumab better benefits PFS and safety. Adding FOLFOX4 was associated with better ORR and OS. The efficacy and safety of an IFL-bevacizumab-FOLFOX4 combination should be given greater weight in future clinical trials, guidelines, and clinical practice.

Keywords: combination therapy, bevacizumab, metastatic colorectal cancer, meta-analysis

\section{Introduction}

Colorectal cancer is one of the most commonly diagnosed cancers and one of the leading causes of cancer-related death worldwide. ${ }^{1}$ Although chemotherapy, radiotherapy, and surgery have been standard treatments for patients with cancers, outcomes of these treatments including the progression-free survival (PFS) of patients are still worrying and far from ideal. ${ }^{2}$ Vascular endothelial growth factor (VEGF) plays a vital role in the regulation of promoting tumor angiogenesis by inducing the proliferation and migration of vascular endothelial cells. ${ }^{3,4}$ In addition to stimulating the formation of blood vessels, especially in the tumor surrounding, VEGF is also critical for the 
enhancement of vascular permeability. ${ }^{5}$ Therefore, targeting VEGF to inhibit tumor growth has become a focus of investigation, including research in the humanized monoclonal antibody bevacizumab. ${ }^{6}$

Bevacizumab, a humanized monoclonal IgG antibody, can prevent the binding of VEGF-A to tyrosine kinase receptors (the VEGFRs) by targeting all isoforms of VEGF-A., Thus, bevacizumab can inhibit the formation of new tumor vasculature and result in the regression of tumor vascularization. In addition, it can decrease elevated interstitial pressure in tumors to help the delivery of concurrently administered other therapy agents. ${ }^{9-11}$ In various murine cancer models, bevacizumab has been proved to exert robust antitumor effects. $^{12-17}$

Recently, blocking angiogenesis has shown promise for metastatic colorectal cancer therapy, and anti-VEGF antibody combination therapy has gained much popularity. However, the survival benefit and safety of anti-VEGF antibody combination therapy remain under debate, and whether the added benefits of each drug outweigh the added risk of toxicity remains uncertain. To the best of our knowledge, few meta-analyses have looked at the survival benefits and safety of different types of anti-VEGF antibody combination therapy.

The aim of the present meta-analysis was to evaluate the clinical value of bevacizumab-based combination therapy and the added benefits and toxicity risk of each drug.

\section{Methods}

\section{Study design and search strategy}

All relevant information of this meta-analysis was identified from published trials that compared bevacizumab-based combination therapy with monotherapy. We searched for the trials based on the following computerized bibliographic databases: PubMed/Medline, Embase, the Cochrane Central Register of Controlled Trials (CENTRAL), and Google Scholar without any language restrictions. February 2018 was the cutoff date. The following keywords were included: combination therapy, bevacizumab, metastatic colorectal cancer, VEGF, oxaliplatin, fluorouracil, and leucovorin (FOLFOX4), fluorouracil/leucovorin (FU/LV), irinotecan, fluorouracil, and leucovorin (IFL), and capecitabine and oxaliplatin (XELOX). In order to find potential publications, we reviewed the reference list of related articles for further analysis.

\section{Selection criteria}

We identified eligible studies according the following five criteria: 1) trials that included patients with metastatic colorectal cancer; 2) Phase II or Phase III trials for cancer; 3 ) an intervention that combined bevacizumab with other treatments, such as FOLFOX4 or IFL; 4) studies that evaluate the clinical value or adverse events (AEs) of treatment; and 5) studies that compare combination therapy and monotherapy.

The exclusion criteria were: 1) the control group is bevacizumab; 2) studies were not related to our research topics or not clinical trials; 3 ) retrospective studies, letters, editorials, expert opinions; and 4) studies that lacked necessary data.

\section{Data extraction}

Three reviewers (RX, CX, and CL) independently extracted data by using a standardized form. We reviewed all the studies and extracted the following information: the first author, published year, intervention in the experimental groups and control groups, drugs and doses in the experimental groups and control groups, PFS, overall survival (OS), overall response rate (ORR), and grade $3 / 4$ treatment-related AEs. ORR was collected directly or calculated according to complete response rate and partial response rate. Initially the reviewers determined ORR and OS. The secondary reviewers assessed PFS and grade 3/4 treatment-related AEs.

\section{Assessment of the study quality and risk of bias}

We used Cochrane Collaboration's tool to evaluate the risk of bias; any controversies were resolved by mutual discussion. Assessment of the study quality was based on the latest 2009 version of the initial Stroke Therapy Academic Industry Roundtable (STAIR) standard. It includes samplesize calculation, inclusion and exclusion criteria, allocation concealment, blinded assessment of outcome, reporting of patients excluded from analysis, reporting potential conflicts of interest, and study funding. All the reviewers assessed the qualities in all included studies. "Unclear" means the quality was not clear.

\section{Statistical analysis}

Statistical analysis, forest plots, sensitivity analysis, and detection of publication bias were performed by Stata/SE 12.0 (Stata Corp, College Station, TX, USA), and we used Review Manager (RevMan5.3; The Nordic Cochrane Center, The Cochrane Collaboration, Copenhagen, Denmark) to assess the risk of bias. In addition, we used an Excel spreadsheet developed by Matthew Sydes and Jayne Tierney of the MRC Clinical Trials Unit (London, UK) to evaluate the $\ln (\mathrm{HR})$ values and $\mathrm{se}(\ln (\mathrm{HR}))$ values. ${ }^{18,19}$ Relative risk (RR) and HRs were used for evaluation. Publication bias was 
assessed by Egger's test and Begg's test. All the analyses (OS, ORR, PFS, and grade 3/4 treatment-related AEs) used a random-effects model ( $\mathrm{M}-\mathrm{H}$ heterogeneity). ${ }^{20}$ In addition, we calculated $95 \%$ CIs for each estimate.

\section{Data availability}

All data generated during and/or analyzed in this study are included in this published article (and its Supplementary materials).

\section{Results}

\section{Search results and study characteristics}

The detailed study selection process is illustrated in Figure S1. A total of 528 potentially relevant studies were retrieved from the initial database search in PubMed/Medline, Embase, CENTRAL, and Google Scholar, of which eleven articles met all inclusion criteria. ${ }^{21-28}$ All the studies evaluated the value of anti-VEGF antibody combination therapy, combining bevacizumab with other therapy. We found several types of combination trials: two combinations of anti-VEGF antibody with FOLFOX4, two combinations of anti-VEGF antibody with FU/LV, two combinations of antiVEGF antibody with IFL, one combination of anti-VEGF antibody with XELOX, and two combinations of anti-VEGF antibody with capecitabine.

A total of 3,424 patients with metastatic colorectal cancer were included in the analysis. The safety analysis included 3,274 of these patients. In total, 1,960 patients received combination therapy, including two combinations of bevacizumab with FOLFOX4 $(n=635)$, two combinations of bevacizumab with FU/LV ( $n=172)$, two combinations of bevacizumab with IFL $(n=516)$, one combination of bevacizumab with XELOX $(n=350)$, and two combinations of bevacizumab with capecitabine $(n=287)$. Patients receiving monotherapy served as the controls $(n=1,464)$. Detailed information of those trials is available in the Table 1.

\section{Efficacy outcomes}

The ORR was reported in eight of the studies. ${ }^{21-28}$ The PFS was reported in seven of the studies. ${ }^{21-25,27,28}$ The OS was reported in seven of the studies..$^{21,23-28}$ The ORR, OS, and PFS of combination therapy were significantly higher than monotherapy (Figure 1). The combination of anti-VEGF antibody and other therapy was associated with significantly higher ORRs than monotherapy (RR: $1.40 ; 95 \%$ CI: 1.10-1.78; $P=0.005$ ) (Figure 1A). Compared with monotherapy, combination therapy significantly prolonged PFS (HR: 0.64; 95\% CI: $0.55-0.73$; $P=0.000$ ) (Figure 1B). In addition, the OS of combination therapy was also significantly higher than monotherapy
(HR: $0.82 ; 95 \%$ CI: 0.73-0.92; $P=0.001$ ) (Figure 1C). There was high heterogeneity in the ORR $\left(I^{2}=79.5 \%\right)$ and PFS $\left(I^{2}=69.8 \%\right)$ analyses, while heterogeneity in OS $\left(I^{2}=45.6 \%\right)$ was moderate. The subgroup analysis of combination type is shown in Figure 2 and Table 2. Detailed heterogeneity analyses are shown in Figure S2. The subgroup analysis of patients is shown in Figure 3. It showed that patients with different sex and baseline Eastern Cooperative Oncology Group performance score have similar PFS improvement in response to bevacizumab-based combination therapy (Figure 3A and B). The subgroup analysis of PFS based on the location of primary disease and previous adjuvant treatment is shown in Figure 3C and D, respectively.

\section{Safety outcomes}

The grade 3/4 treatment-related AEs of anti-VEGF antibody combination therapy were reported in six studies. ${ }^{21-25,28}$ Analysis shows significantly high rate of grade 3/4 AEs in combination therapies (RR: 1.27 ; 95\% CI: $1.15-1.41$; $P=0.000)$ (Figure 4A), with high heterogeneity $\left(I^{2}=60.5 \%\right)$. Tests of heterogeneity and subgroup analyses by type of combination are shown in Figure 4B and Table 3. Combination of anti-VEGF antibody and capecitabine showed the highest rate of grade 3/4 treatment-related AEs (RR: 1.89; 95\% CI: 1.31-2.73; $P=0.000$ ), while combination of antiVEGF antibody and IFL showed the lowest rate of grade 3/4 treatment-related AEs (RR: 1.15; 95\% CI: 1.07-1.23; $P=0.000$ ) (Figure 4B).

\section{Sensitivity analysis and publication bias}

Detailed sensitivity analyses are shown in Figure 5. All sensitivity analysis associated with the meta-analyses performed in this study indicated a stable results, and no sensitivity analysis shows positive results. Our publication bias was based on both Begg's test and Egger's test. In Begg's test, the $P$-values were 0.386 for ORR, 0.063 for grade $3 / 4$ treatmentrelated AEs, 0.474 for PFS, and 0.858 for OS. In Egger's test, the $P$-values were 0.116 for ORR, 0.052 for grade $3 / 4$ treatment-related AEs, 0.412 for PFS, and 0.968 for OS. Begg graphs are shown in Figure S3, and Egger graphs are shown in Figure S4. Details on the risk of bias in 14 studies are illustrated in Figure S5.

\section{Discussion}

To the best of our knowledge, this is the first metaanalysis with randomized controlled trials to evaluate the added benefits and toxicity of each drug in bevacizumabbased combination therapy. The current trials have some 


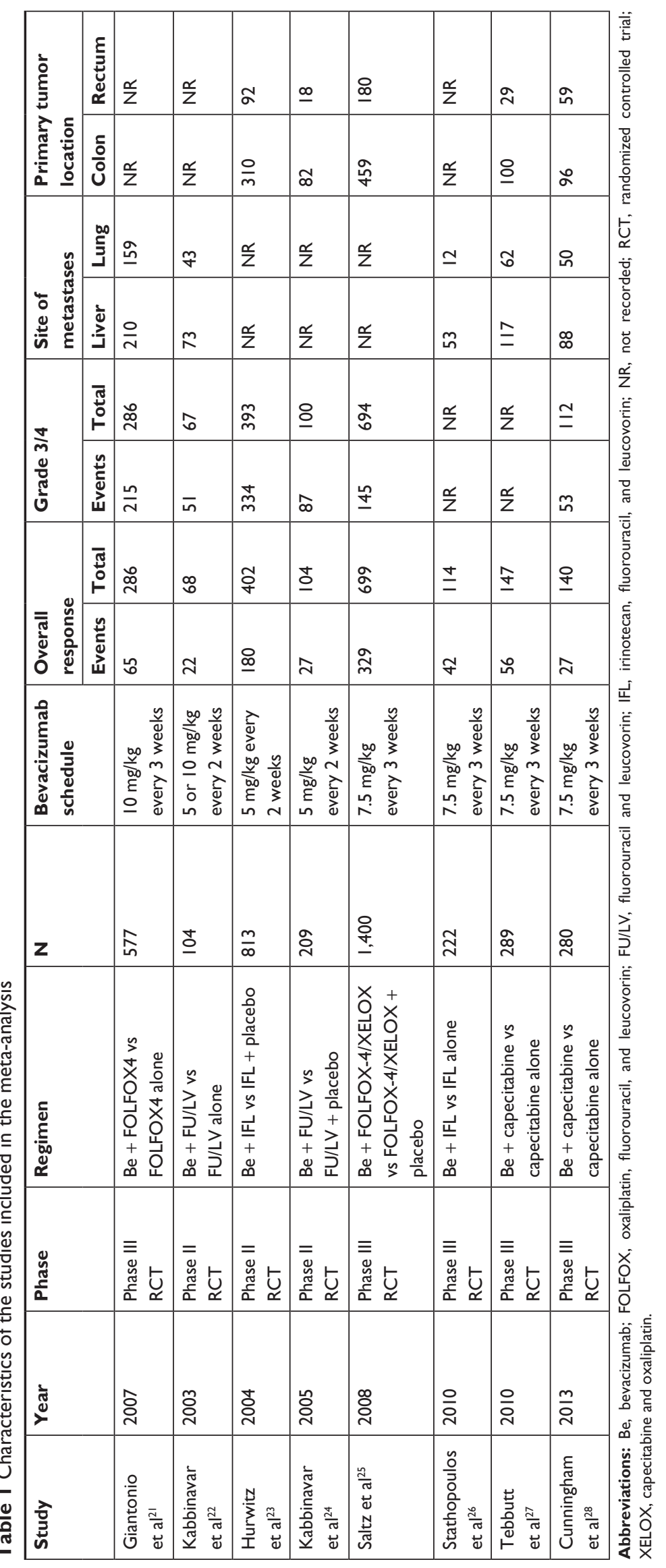


limitations, but we think the outcomes can still provide insights into anti-VEGF antibody combination therapy. Outcomes of studies on anti-VEGF antibody combination therapy including FOLFOX4, FU/LV, IFL, XELOX, and capecitabine have been published, but efficacy and safety of combination therapy are still under debate. Some studies reported that anti-VEGF antibody combination therapy did not significantly improve PFS or OS, but it did increase toxicity. ${ }^{21,26}$ Therefore, we performed this meta-analysis to evaluate the value and toxic effects of anti-VEGF antibody combination therapy; in addition, subgroup analyses were warranted to evaluate the optimal type of combination.

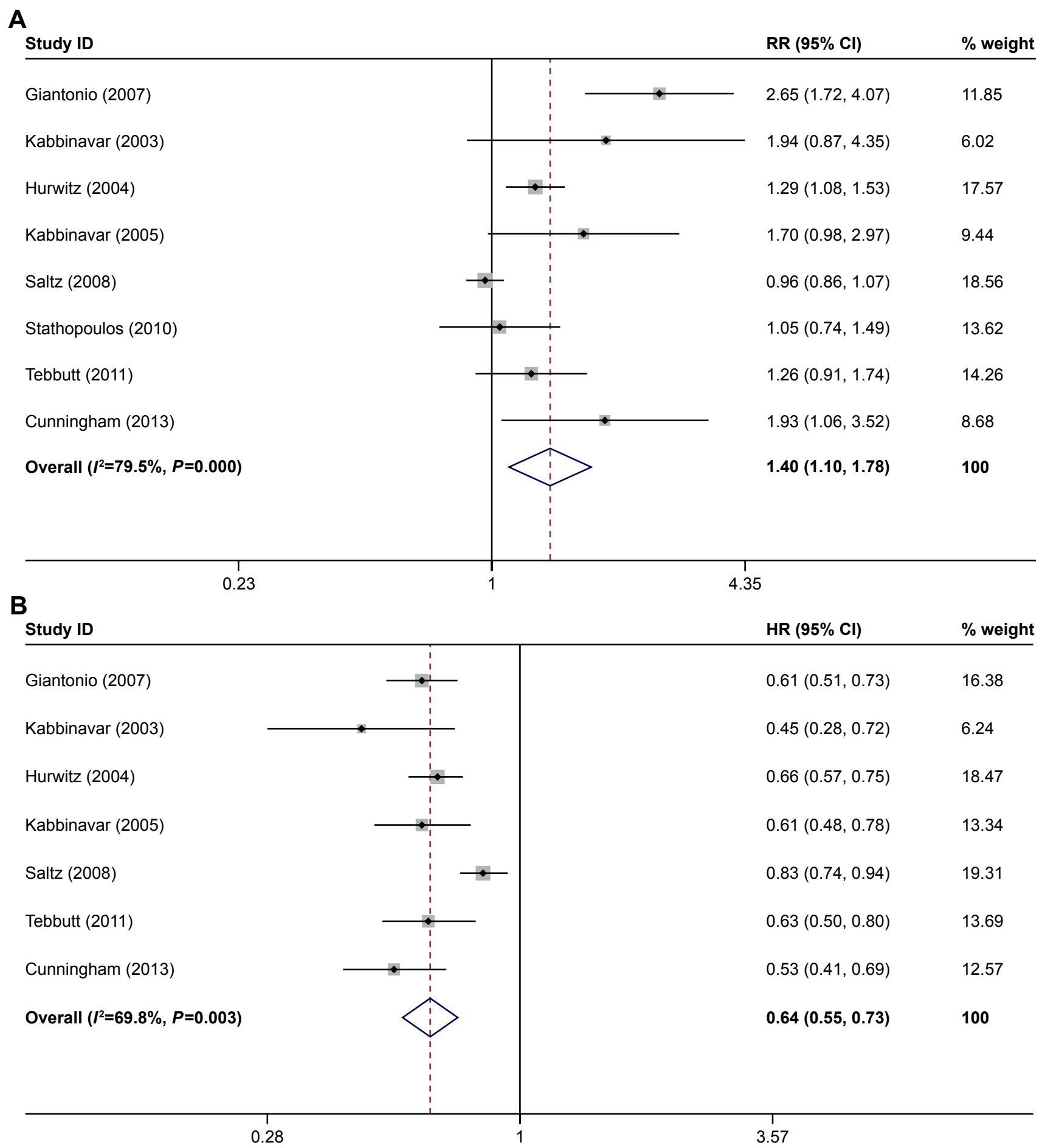

Figure I (Continued) 


\section{C}

\section{Study ID}

$\mathrm{HR}(95 \% \mathrm{Cl})$

$\%$ weight

Giantonio (2007)

Hurwitz (2004)

Kabbinavar (2005)

Saltz (2008)

Stathopoulos (2010)

Tebbutt (2011)

Cunningham (2013)

Overall $\left(I^{2}=45.6 \%, P=0.088\right)$

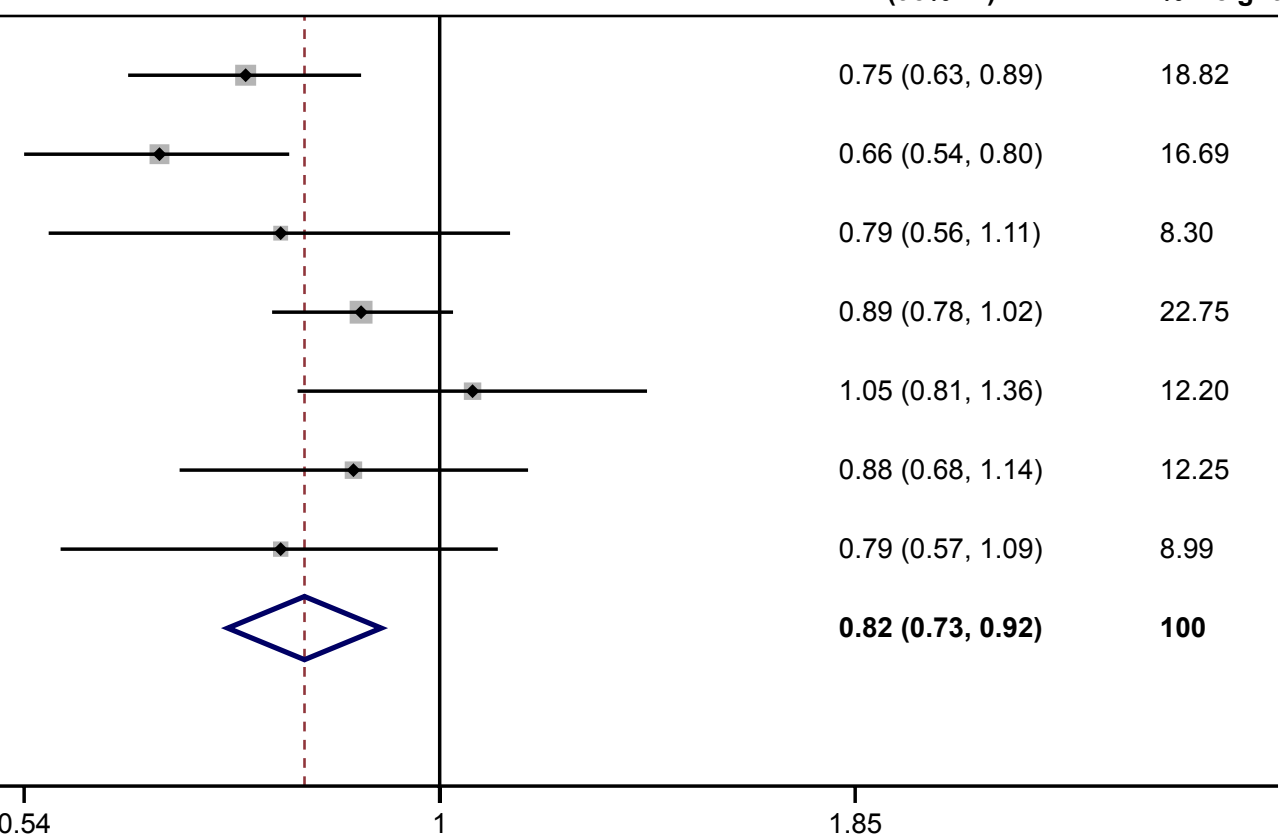

Figure I Meta-analysis of (A) ORR, (B) PFS, and (C) OS.

Note: Weights are from random-effects analysis.

Abbreviations: ORR, overall response rate; PFS, progression-free survival; OS, overall survival.

\section{A}

Study ID

$\mathrm{RR}(95 \% \mathrm{Cl})$

$\%$ weight

FOLFOX4

Giantonio (2007)

Subtotal $\left(I^{2}=. \%, P=.\right)$

FU/LV

Kabbinavar (2003)

Kabbinavar (2005)

Subtotal $\left(I^{2}=0.0 \%, P=0.794\right)$

IFL

Hurwitz (2004)

Stathopoulos (2010)

Subtotal $\left(I^{2}=6.6 \%, P=0.301\right)$

Capecitabine

Tebbutt (2011)

Cunningham (2013)

Subtotal $\left(I^{2}=34.8 \%, P=0.216\right)$

Overall $\left(I^{2}=59.7 \%, P=0.021\right)$

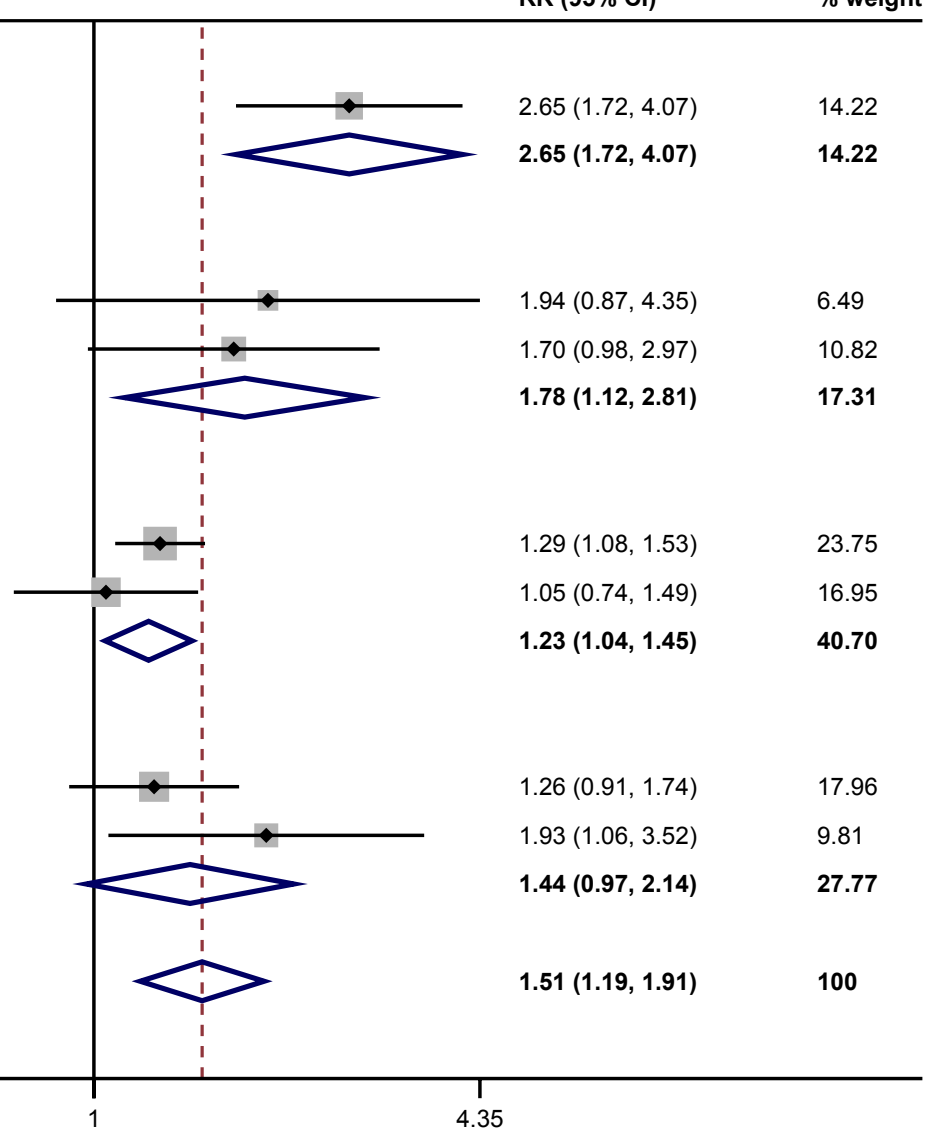

Figure 2 (Continued) 
B

Study ID

HR $(95 \% \mathrm{Cl})$

$\%$ weight

FOLFOX4

Giantonio (2007)

Saltz(a) (2008)

Subtotal $\left(I^{2}=87.1 \%, P=0.005\right)$

FU/LV

Kabbinavar (2003)

Kabbinavar (2005)

Subtotal $\left(I^{2}=20.7 \%, P=0.261\right)$

IFL

Hurwitz (2004)

Subtotal $\left(I^{2}=. \%, P=.\right)$

XELOX

Saltz(b) (2008)

Subtotal $\left(I^{2}=. \%, P=\right.$. $)$

Capecitabine

Tebbutt (2011)

Cunningham (2013)

Subtotal $\left(I^{2}=0.0 \%, P=0.334\right)$

Overall $\left(I^{2}=60.5 \%, P=0.013\right)$

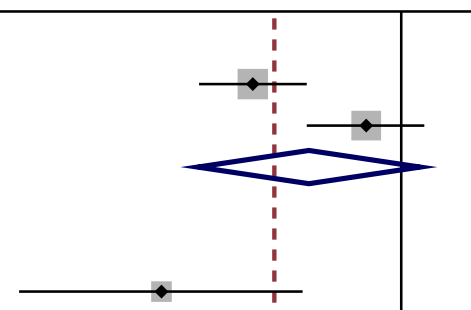

$0.61(0.51,0.73)$

14.92

$0.89(0.73,1.08)$

14.05

$0.74(0.51,1.06)$

28.96

$0.45(0.28,0.72)$

5.12

$0.61(0.48,0.78)$

11.76

$0.56(0.43,0.73)$

16.88

Overall $\left(1^{2}=60.5 \%, P=0.013\right)$

C

\begin{tabular}{|c|c|c|}
\hline Study ID & $\mathrm{HR}(95 \% \mathrm{Cl})$ & $\%$ weigh \\
\hline FOLFOX4 & & \\
\hline Giantonio (2007) & $0.75(0.63,0.89)$ & 23.82 \\
\hline Subtotal $\left(I^{2}=. \%, P=.\right)$ & $0.75(0.63,0.89)$ & 23.82 \\
\hline IFL & & \\
\hline Hurwitz (2004) & $0.66(0.54,0.80)$ & 21.33 \\
\hline Stathopoulos (2010) & $1.05(0.81,1.36)$ & 15.93 \\
\hline Subtotal $\left(I^{2}=87.2 \%, P=0.005\right)$ & $0.83(0.52,1.30)$ & 37.26 \\
\hline FU/LV & & \\
\hline Kabbinavar (2005) & $0.79(0.56,1.11)$ & 11.03 \\
\hline Subtotal $\left(I^{2}=. \%, P=.\right)$ & $0.79(0.56,1.11)$ & 11.03 \\
\hline Capecitabine & & \\
\hline Tebbutt (2011) & $0.88(0.68,1.14)$ & 15.98 \\
\hline Cunningham (2013) & $0.79(0.57,1.09)$ & 11.91 \\
\hline Subtotal $\left(I^{2}=0.0 \%, P=0.610\right)$ & $0.84(0.69,1.03)$ & 27.89 \\
\hline Overall $\left(I^{2}=43.5 \%, P=0.115\right)$ & $0.80(0.70,0.91)$ & 100 \\
\hline
\end{tabular}

Figure 2 Subgroup analysis of (A) ORR, (B) PFS, and (C) OS by type of combination.

Note: Weights are from random-effects analysis.

Abbreviations: ORR, overall response rate; PFS, progression-free survival; OS, overall survival; FU/LV, fluorouracil/leucovorin; IFL, irinotecan, fluorouracil, and leucovorin. 
Table 2 Subgroup analysis by type of bevacizumab-based combination

\begin{tabular}{|c|c|c|c|c|c|}
\hline & Heterogeneity & $d f$ & $P$-value & $I^{2}$ & $\tau^{2}$ \\
\hline \multicolumn{6}{|l|}{ ORR } \\
\hline Bevacizumab + FU/LV vs FU/LV & 0.07 & 1 & 0.794 & $0.0 \%$ & 0.0000 \\
\hline Bevacizumab + IFL vs IFL & 1.07 & 1 & 0.301 & $6.6 \%$ & 0.0014 \\
\hline Bevacizumab + capecitabine vs capecitabine & 1.53 & 1 & 0.216 & $34.8 \%$ & 0.0324 \\
\hline Bevacizumab + FOLFOX4 vs FOLFOX4 & 0.00 & 0 & NA & NA & 0.0530 \\
\hline \multicolumn{6}{|l|}{ OS } \\
\hline Bevacizumab + FU/LV vs FU/LV & 0.00 & 0 & NA & NA & 0.0000 \\
\hline Bevacizumab + IFL vs IFL & 7.83 & 1 & 0.005 & $87.2 \%$ & 0.0940 \\
\hline Bevacizumab + capecitabine vs capecitabine & 0.26 & 1 & 0.610 & $0.0 \%$ & 0.0000 \\
\hline Bevacizumab + FOLFOX4 vs FOLFOX4 & 0.00 & 0 & NA & NA & 0.0000 \\
\hline \multicolumn{6}{|l|}{ PFS } \\
\hline Bevacizumab + FU/LV vs FU/LV & 1.26 & 1 & 0.261 & $20.7 \%$ & 0.0096 \\
\hline Bevacizumab + IFL vs IFL & 0.00 & 0 & NA & NA & 0.0000 \\
\hline Bevacizumab + XELOX vs XELOX & 0.00 & 0 & NA & NA & 0.0000 \\
\hline Bevacizumab + capecitabine vs capecitabine & 0.93 & 1 & 0.334 & $0.0 \%$ & 0.0000 \\
\hline Bevacizumab + FOLFOX4 vs FOLFOX4 & 7.78 & I & 0.005 & $87.1 \%$ & 0.0622 \\
\hline
\end{tabular}

Abbreviations: $\mathrm{df}$, degrees of freedom; FOLFOX4, oxaliplatin, fluorouracil, and leucovorin; FU/LV, fluorouracil and leucovorin; IFL, irinotecan, fluorouracil, and leucovorin; NA, not available; ORR, overall response rate; OS, overall survival; PFS, progression-free survival; XELOX, capecitabine and oxaliplatin.

\section{Main findings}

The pooled analyses showed that anti-VEGF antibody combination therapy led to significantly improved ORR, OS, and PFS in comparison with monotherapy. However, most combinations were associated with higher rate of grade $3 / 4$ treatment-related AEs.

After all bevacizumab-based combination therapies were evaluated, we found that combining bevacizumab with FOLFOX4 yielded the best ORR while the combination of bevacizumab and IFL was associated with only slight ORR improvement. Improvements in PFS and OS were documented in all combinations. Combining bevacizumab with FOLFOX4 and bevacizumab with IFL led to improved OS and PFS significantly.

In terms of toxicity, we found that combining bevacizumab with capecitabine led to the highest rate of grade $3 / 4$ treatment-related AEs, and combinations of bevacizumab with IFL showed the lowest rate of grade 3/4 treatment-related

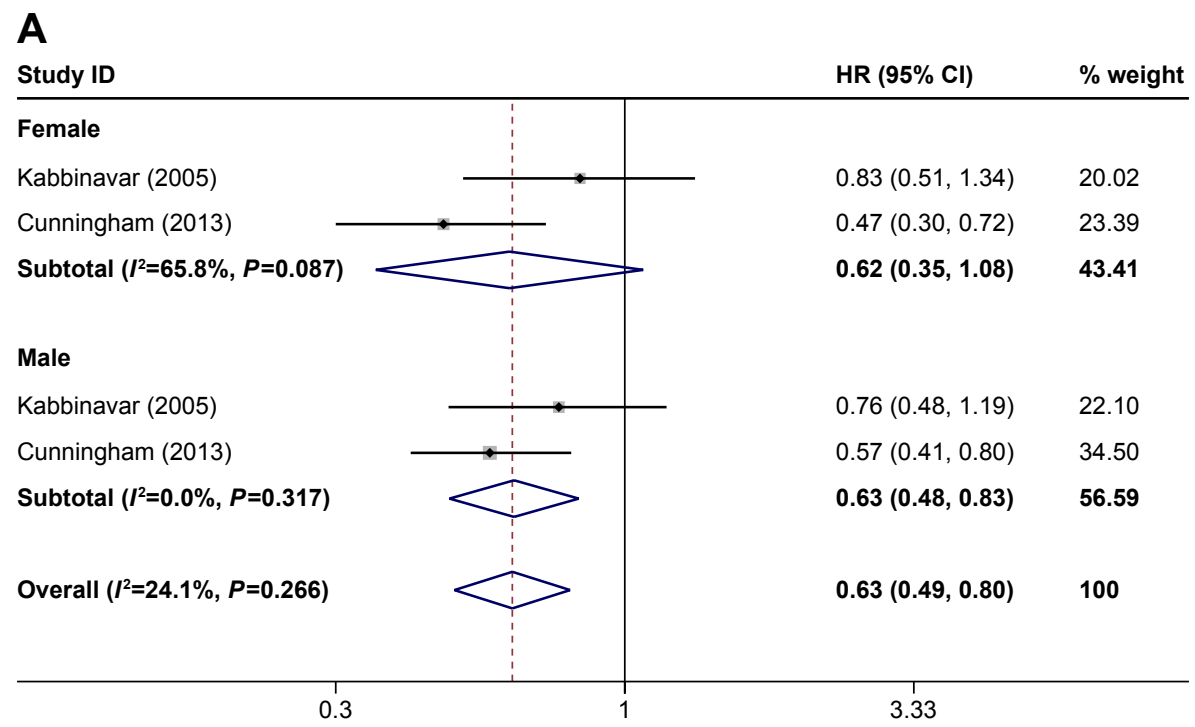

Figure 3 (Continued) 
B

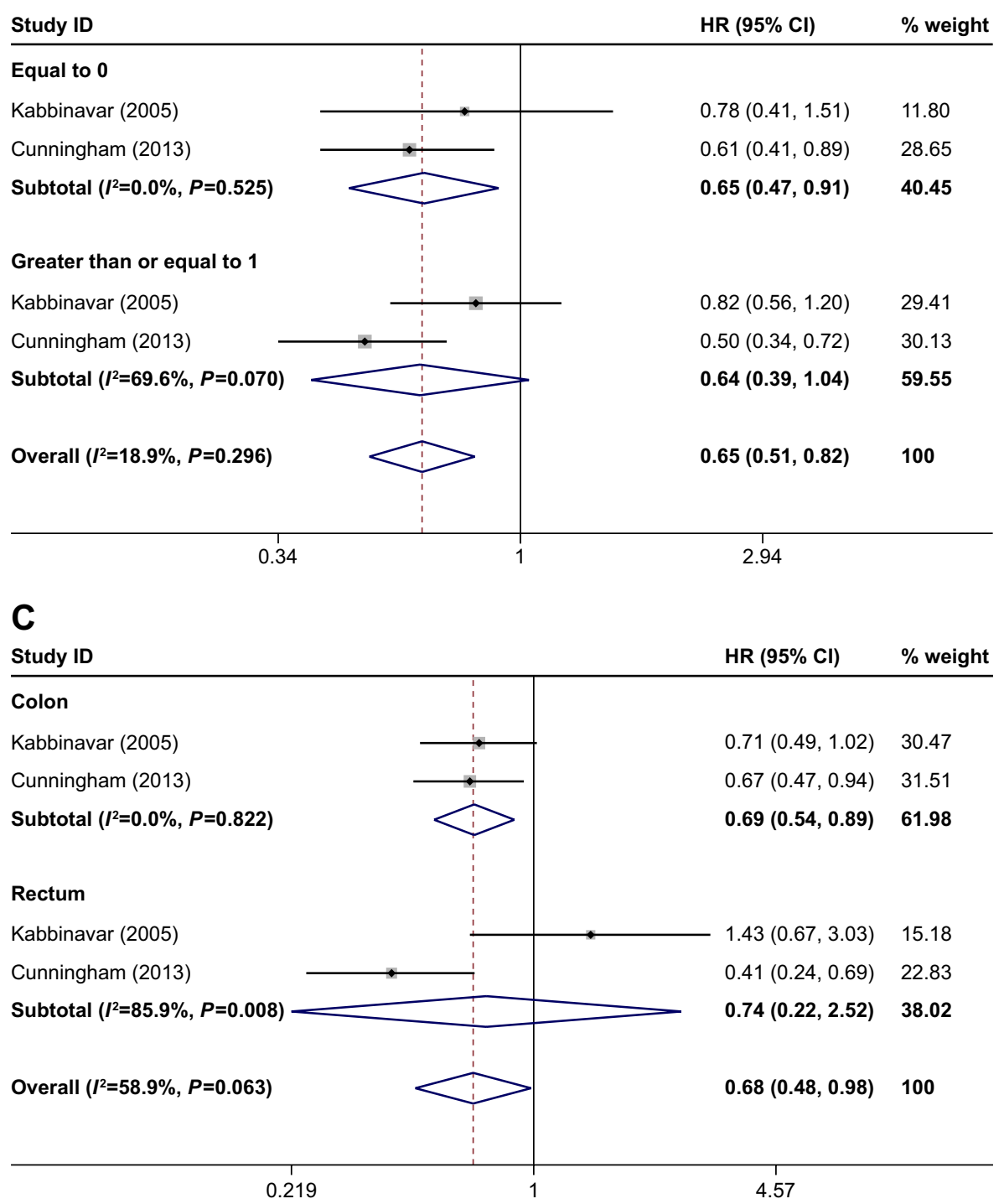

D

Study ID

$\mathrm{HR}(95 \% \mathrm{Cl})$

$\%$ weight

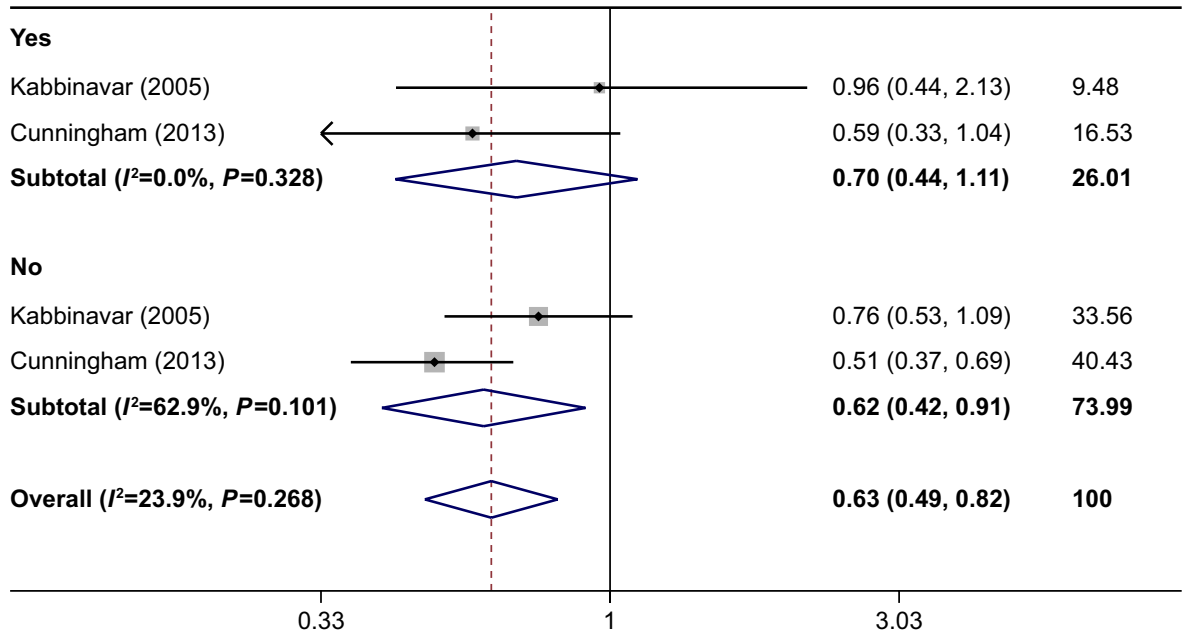

Figure 3 Subgroup analysis of PFS based on (A) sex, (B) baseline ECOG performance score, (C) location of primary disease, and (D) previous adjuvant treatment. Note: Weights are from random-effects analysis.

Abbreviation: PFS, progression-free survival. 
A

Study ID

RR $(95 \% \mathrm{Cl})$

$\%$ weight

Giantonio (2007)

Kabbinavar (2003)

Hurwitz (2004)

Kabbinavar (2005)

Saltz (2008)

Cunningham (2013)

Overall $\left(I^{2}=58.8 \%, P=0.033\right)$

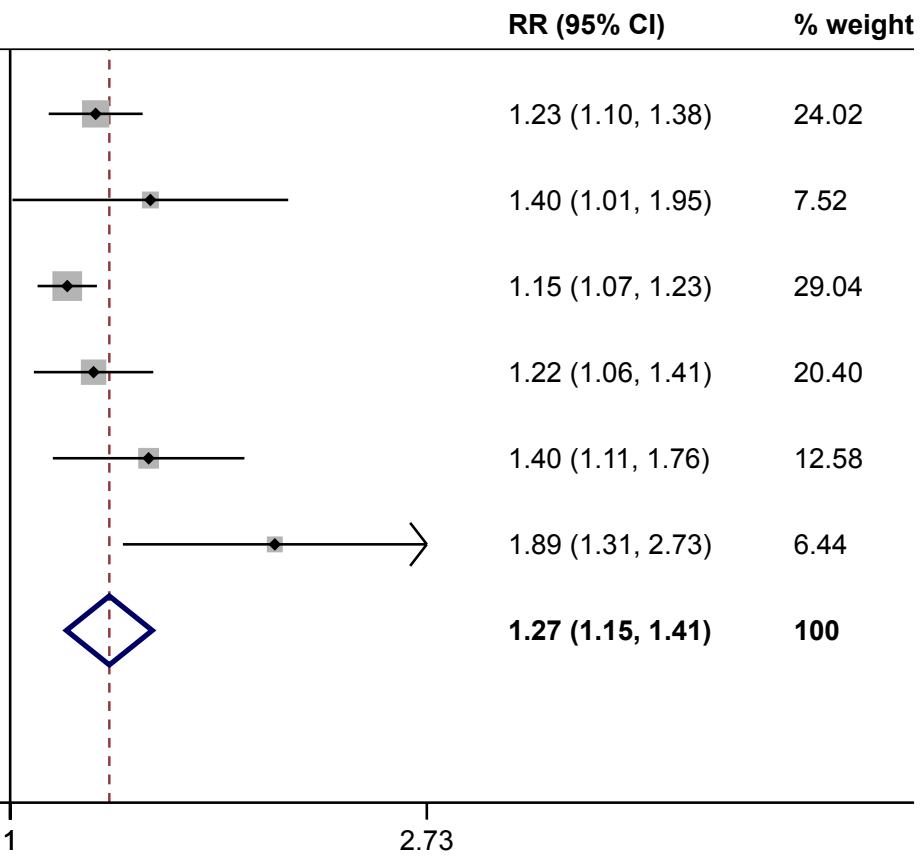

B

0.366

2.73

\section{Study ID}

FOLFOX4

Giantonio (2007)

Subtotal $\left(I^{2}=. \%, P=.\right)$

\section{FU/LV}

Kabbinavar (2003)

Kabbinavar (2005)

Subtotal $\left(I^{2}=0.0 \%, P=0.438\right)$

IFL

Hurwitz (2004)

Subtotal $\left(I^{2}=. \%, P=.\right)$

\section{Capecitabine}

Cunningham (2013)

Subtotal $\left(I^{2}=. \%, P=.\right)$

Overall $\left(I^{2}=57.5 \%, P=0.052\right)$

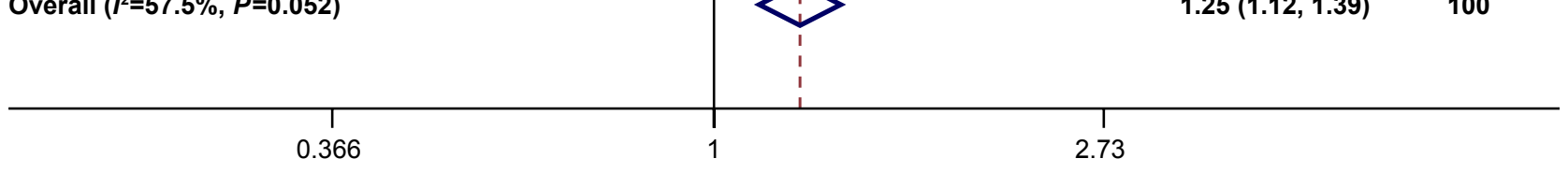

Figure 4 (A) Meta-analysis of grade 3/4 treatment-related adverse events; (B) subgroup analysis of grade 3/4 treatment-related adverse events by type of combination. Note: Weights are from random-effects analysis.

Abbreviations: FU/LV, fluorouracil/leucovorin; IFL, irinotecan, fluorouracil, and leucovorin. 
Table 3 Grade 3/4 treatment-related adverse events, analyzed by type of bevacizumab-based combination

\begin{tabular}{|l|l|l|l|l|l|}
\hline G3/4 trAEs & Heterogeneity & $\mathbf{d f}$ & $\boldsymbol{P}$-value & $\mathbf{I}^{\mathbf{2}}$ & $\tau^{\mathbf{2}}$ \\
\hline Bevacizumab + FOLFOX4 vs FOLFOX4 & 0.00 & 0 & NA & NA & 0.0000 \\
\hline Bevacizumab + FU/LV vs FU/LV & 0.60 & $\mathrm{I}$ & 0.438 & $0.0 \%$ & 0.0000 \\
\hline Bevacizumab + IFL vs IFL & 0.00 & 0 & NA & NA & 0.0000 \\
\hline Bevacizumab + capecitabine vs capecitabine & 0.00 & 0 & NA & NA & 0.0000 \\
\hline
\end{tabular}

Abbreviations: $d f$, degrees of freedom; FOLFOX4, oxaliplatin, fluorouracil, and leucovorin; FU/LV, fluorouracil and leucovorin; G3/4 trAEs, grade 3/4 treatment-related adverse events; IFL, irinotecan, fluorouracil, and leucovorin; NA, not available.

AEs. Thus, combination of bevacizumab with IFL is the best choice in safety.

This meta-analysis shows that the addition of IFL to bevacizumab better benefits PFS and safety. Adding FOLFOX4 was associated with better ORR and OS. Combining FOLFOX4, bevacizumab, and IFL may be a new effective strategy of antiVEGF antibody combination therapy for patients with metastatic colorectal cancer. The efficacy and safety of anti-VEGF antibody combination therapy should be further investigated for its potential to extend the clinical success.
A

Meta-analysis estimates, given named study is omitted

I Lower Cl limit o Estimate I Upper Cl limit

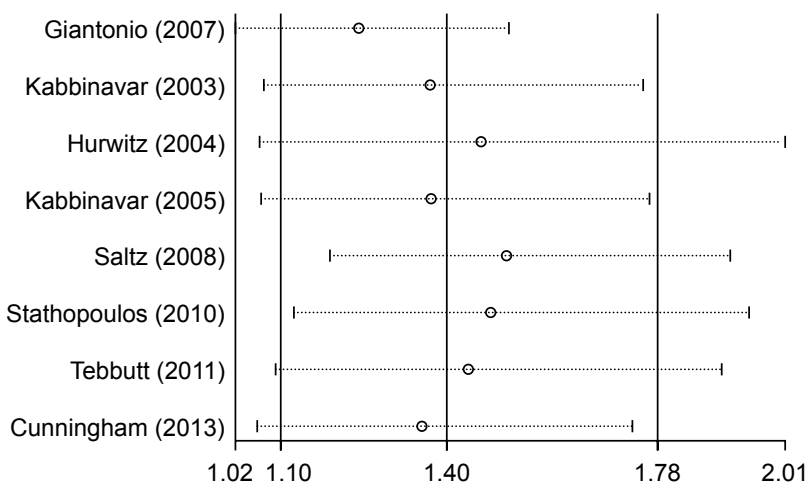

C

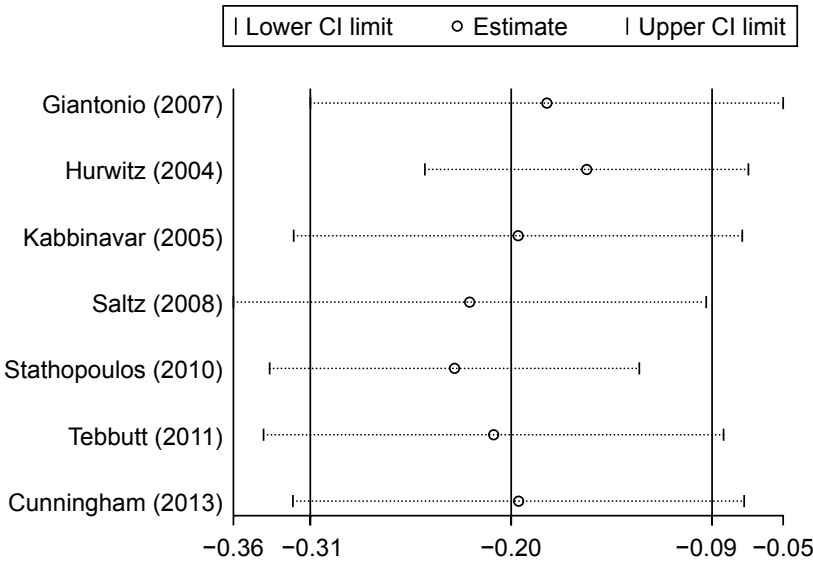

B

Meta-analysis estimates, given named study is omitted

I Lower Cl limit $\quad$ O Estimate I Upper Cl limit

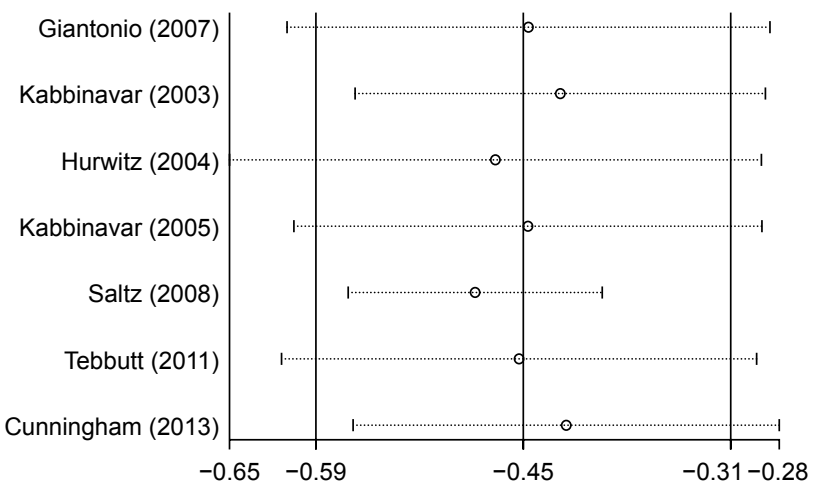

D

Meta-analysis estimates, given named study is omitted

I Lower Cl limit o Estimate I Upper Cl limit

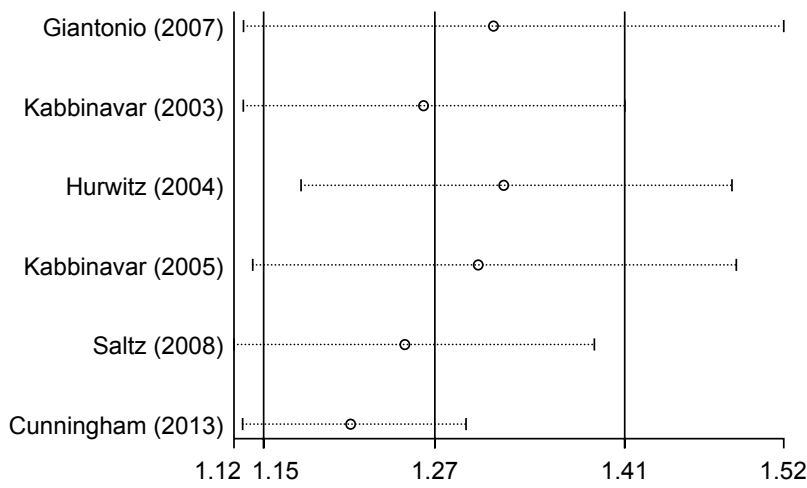

Figure 5 Sensitivity analysis of (A) ORR; (B) PFS; (C) OS; and (D) grade 3/4 treatment-related adverse events. Bars $=95 \%$ Cl. Results symbolized by omitting each study in turn.

Abbreviations: ORR, overall response rate; PFS, progression-free survival; OS, overall survival. 


\section{Comparison with previous meta-analyses}

Some previous studies have assessed the efficacy of bevacizumab. ${ }^{29-32}$ Most of them evaluate only the efficacy of adding chemotherapy drugs to bevacizumab, ${ }^{29-31}$ and one of them evaluate only the efficacy of safety of combination of bevacizumab and erlotinib..$^{32}$ To the best of our knowledge, no other meta-analysis evaluated the benefits against toxicity of each drug added in the anti-VEGF antibody combination. Compared with previous meta-analyses, our current study identifies the combination of FOLFOX4, bevacizumab, and IFL as a novel type of anti-VEGF antibody combination, which may provide a new strategy for the therapy of metastatic colorectal cancer.

\section{Limitations}

This study has some limitations. Some studies have reported only short-term follow-up and lack of long-term outcomes. In addition, the ORR, PFS, and grade 3/4 treatment-related AEs were not available in some of the reports. Therefore, more study investigation should be prospectively planned and performed in the future.

\section{Conclusion}

This meta-analysis showed that the addition of IFL to bevacizumab better benefits PFS and safety. Adding FOLFOX4 was associated with better ORR and OS. The efficacy and safety of an IFL-bevacizumab-FOLFOX4 combination should be investigated in the future.

\section{Summary}

- This meta-analysis evaluated the safety and efficacy of bevacizumab-based combination therapy and found a more beneficial combination.

- Bevacizumab-based combination therapy can significantly improve ORR, PFS, and OS, but combination therapy also showed high toxicity.

- Adding IFL to bevacizumab better benefits PFS and safety.

- Adding FOLFOX4 to bevacizumab was associated with better ORR and OS.

- Combining FOLFOX4, bevacizumab, and IFL may be a new effective strategy of anti-VEGF antibody combination therapy for patients with metastatic colorectal cancer in the future.

\section{Acknowledgments}

The authors thank the authors of the included trials for providing the data. The authors received no specific funding for this work.

\section{Author contributions}

$\mathrm{RX}$ and $\mathrm{JZ}$ were responsible for concept and study design. $\mathrm{RX}, \mathrm{CX}, \mathrm{CC}$, and JZ were responsible for publication retrieve and data extraction. RX, CX, and CL were responsible for statistical analyses. All authors contributed to data analysis, drafting and revising the article, gave final approval of the version to be published, and agree to be accountable for all aspects of the work.

\section{Disclosure}

The authors report no conflicts of interest in this work.

\section{References}

1. Siegel RL, Miller KD, Fedewa SA, et al. Colorectal cancer statistics, 2017. CA Cancer J Clin. 2017;67(3):177-193.

2. Devita VT, Chu E. A history of cancer chemotherapy. Cancer Res. 2008;68(21):8643-8653.

3. Ferrara N. Vascular endothelial growth factor: basic science and clinical progress. Endocr Rev. 2004;25(4):581-611.

4. Ferrara N. Vascular endothelial growth factor as a target for anticancer therapy. Oncologist. 2004;9(Suppl 1):2-10.

5. Rak JW, St Croix BD, Kerbel RS. Consequences of angiogenesis for tumor progression, metastasis and cancer therapy. Anticancer Drugs. 1995;6(1):3-18.

6. Ferrara N, Hillan KJ, Gerber HP, Novotny W. Discovery and development of bevacizumab, an anti-VEGF antibody for treating cancer. Nat Rev Drug Discov. 2004;3(5):391-400.

7. Gerber HP, Ferrara N. Pharmacology and pharmacodynamics of bevacizumab as monotherapy or in combination with cytotoxic therapy in preclinical studies. Cancer Res. 2005;65(3):671-680.

8. Lee JJ, Chu E. Sequencing of antiangiogenic agents in the treatment of metastatic colorectal cancer. Clin Colorectal Cancer. 2014;13(3): 135-144.

9. Xu L, Duda DG, di Tomaso E, et al. Direct evidence that bevacizumab, an anti-VEGF antibody, up-regulates SDF1alpha, CXCR4, CXCL6, and neuropilin 1 in tumors from patients with rectal cancer. Cancer Res. 2009; 69(20):7905-7910

10. Jain RK. Normalizing tumor vasculature with anti-angiogenic therapy: a new paradigm for combination therapy. Nat Med. 2001;7(9): 987-989.

11. Kerbel RS. Antiangiogenic therapy: a universal chemosensitization strategy for cancer? Science. 2006;312(5777):1171-1175.

12. Kolinsky K, Shen BQ, Zhang YE, et al. In vivo activity of novel capecitabine regimens alone and with bevacizumab and oxaliplatin in colorectal cancer xenograft models. Mol Cancer Ther. 2009;8(1):75-82.

13. Kolinsky K, Zhang YE, Dugan U, Heimbrook D, Packman K, Higgins B. Novel regimens of capecitabine alone and combined with irinotecan and bevacizumab in colorectal cancer xenografts. Anticancer Res. 2009; 29(1):91-98.

14. Yanagisawa M, Yorozu K, Kurasawa M, et al. Bevacizumab improves the delivery and efficacy of paclitaxel. Anticancer Drugs. 2010;21(7): 687-694.

15. Yanagisawa M, Fujimoto-Ouchi K, Yorozu K, Yamashita Y, Mori K. Antitumor activity of bevacizumab in combination with capecitabine and oxaliplatin in human colorectal cancer xenograft models. Oncol Rep. 2009;22(2):241-247.

16. Mabuchi S, Terai Y, Morishige K, et al. Maintenance treatment with bevacizumab prolongs survival in an in vivo ovarian cancer model. Clin Cancer Res. 2008;14(23):7781-7789.

17. Oliva P, Decio A, Castiglioni V, et al. Cisplatin plus paclitaxel and maintenance of bevacizumab on tumour progression, dissemination, and survival of ovarian carcinoma xenograft models. Br J Cancer. 2012; 107(2):360-369. 
18. Parmar MK, Torri V, Stewart L. Extracting summary statistics to perform meta-analyses of the published literature for survival endpoints. Stat Med. 1998;17(24):2815-2834.

19. Williamson PR, Smith CT, Hutton JL, Marson AG. Aggregate data meta-analysis with time-to-event outcomes. Stat Med. 2002;21(22): 3337-3351.

20. Dersimonian R, Laird N. Meta-analysis in clinical trials. Control Clin Trials. 1986;7(3):177-188.

21. Giantonio BJ, Catalano PJ, Meropol NJ, et al. Bevacizumab in combination with oxaliplatin, fluorouracil, and leucovorin (FOLFOX4) for previously treated metastatic colorectal cancer: results from the Eastern Cooperative Oncology Group Study E3200. J Clin Oncol. 2007; 25(12):1539-1544.

22. Kabbinavar F, Hurwitz HI, Fehrenbacher L, et al. Phase II, randomized trial comparing bevacizumab plus fluorouracil (FU)/leucovorin (LV) with FU/LV alone in patients with metastatic colorectal cancer. $J$ Clin Oncol. 2003;21(1):60-65.

23. Hurwitz H, Fehrenbacher L, Novotny W, et al. Bevacizumab plus irinotecan, fluorouracil, and leucovorin for metastatic colorectal cancer. N Engl J Med. 2004;350(23):2335-2342.

24. Kabbinavar FF, Schulz J, McCleod M, et al. Addition of bevacizumab to bolus fluorouracil and leucovorin in first-line metastatic colorectal cancer: results of a randomized phase II trial. J Clin Oncol. 2005;23(16): 3697-3705.

25. Saltz LB, Clarke S, Díaz-Rubio E, et al. Bevacizumab in combination with oxaliplatin-based chemotherapy as first-line therapy in metastatic colorectal cancer: a randomized phase III study. J Clin Oncol. 2008; 26(12):2013-2019.
26. Stathopoulos GP, Batziou C, Trafalis D, et al. Treatment of colorectal cancer with and without bevacizumab: a phase III study. Oncology. 2010; 78(5-6):376-381.

27. Tebbutt NC, Wilson K, Gebski VJ, et al. Capecitabine, bevacizumab, and mitomycin in first-line treatment of metastatic colorectal cancer: results of the Australasian Gastrointestinal Trials Group Randomized Phase III MAX Study. J Clin Oncol. 2010;28(19):3191-3198.

28. Cunningham D, Lang I, Marcuello E, et al. Bevacizumab plus capecitabine versus capecitabine alone in elderly patients with previously untreated metastatic colorectal cancer (AVEX): an open-label, randomised phase 3 trial. Lancet Oncol. 2013;14(11):1077-1085.

29. Zhang G, Zhou X, Lin C. Efficacy of chemotherapy plus bevacizumab as first-line therapy in patients with metastatic colorectal cancer: a metaanalysis and up-date. Int J Clin Exp Med. 2015;8(1):1434-1445.

30. Chen K, Gong Y, Zhang Q, Shen Y, Zhou T. Efficacy and safety of addition of bevacizumab to FOLFIRI or irinotecan/bolus 5-FU/LV (IFL) in patients with metastatic colorectal cancer: A meta-analysis. Medicine. 2016;95(46):e5221.

31. Cao Y, Tan A, Gao F, Liu L, Liao C, Mo Z. A meta-analysis of randomized controlled trials comparing chemotherapy plus bevacizumab with chemotherapy alone in metastatic colorectal cancer. Int J Colorectal Dis. 2009;24(6):677-685.

32. Xu W, Gong Y, Kuang M, et al. Survival Benefit and Safety of Bevacizumab in Combination with Erlotinib as Maintenance Therapy in Patients with Metastatic Colorectal Cancer: A Meta-Analysis. Clin Drug Investig. 2017;37(2):155-165. 


\section{Supplementary materials}

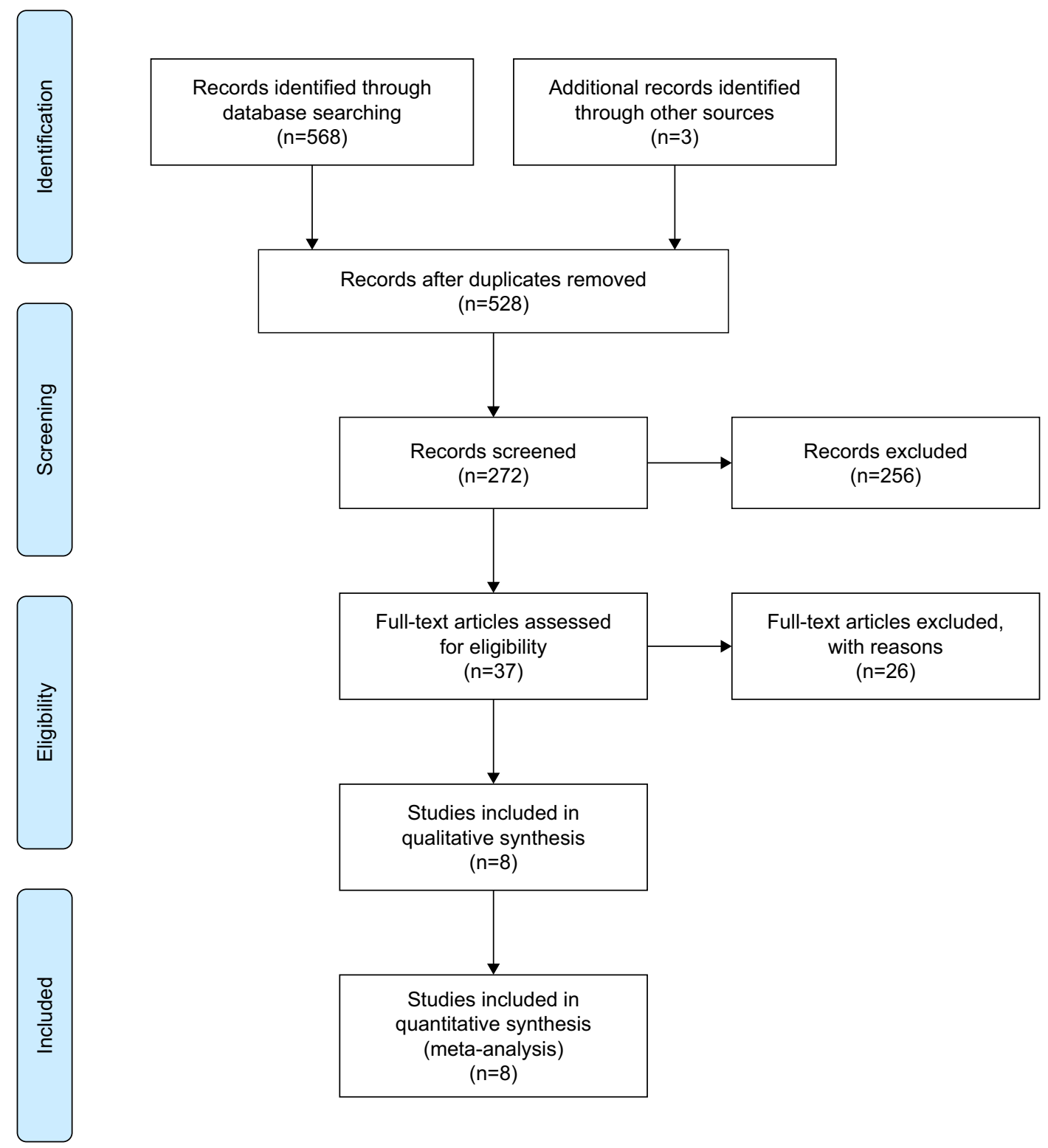

Figure SI The flow diagram of the study selection process. 
A

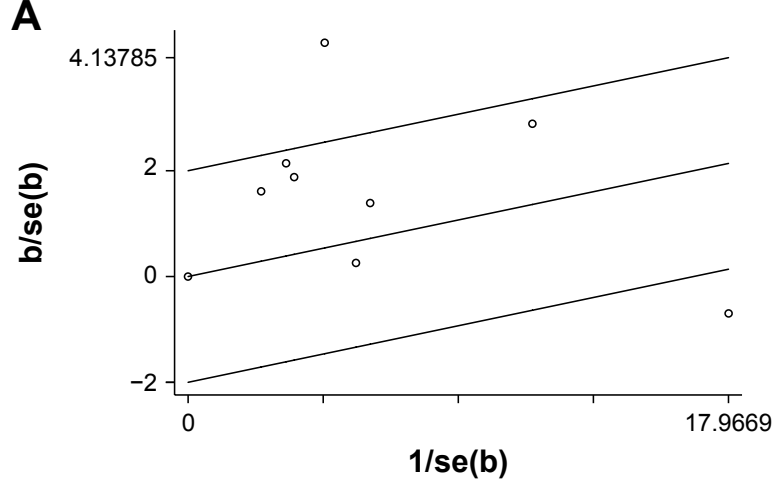

C

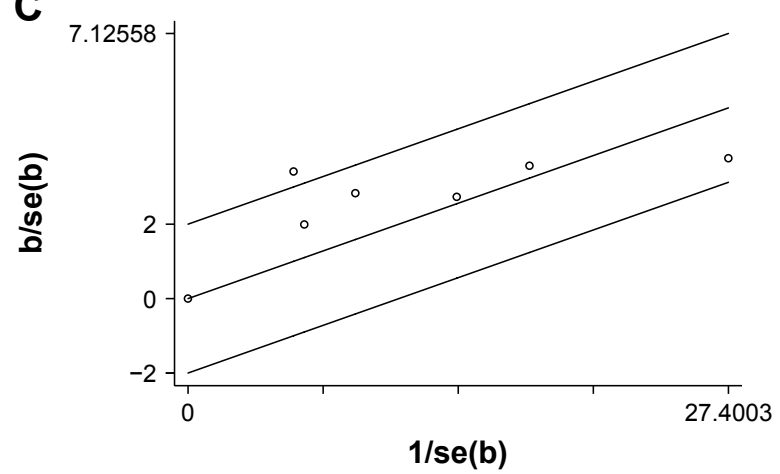

B

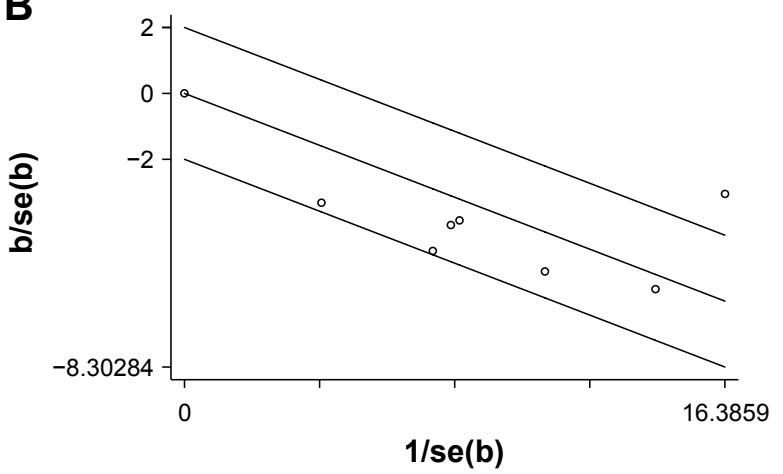

D

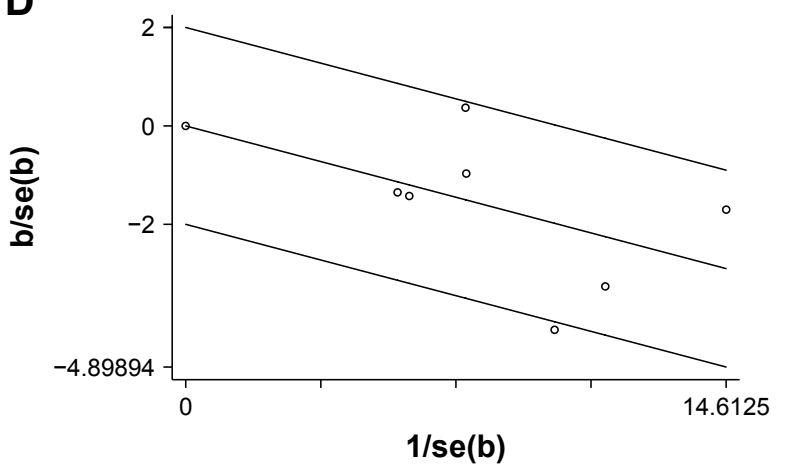

$\circ \mathrm{b} / \mathrm{se}(\mathrm{b}) \longrightarrow$ Fitted values

Figure S2 Heterogeneity analysis. (A) Overall response rate (ORR); (B) progression-free survival (PFS); (C) overall survival (OS); (D) grade 3/4 treatment-related adverse events.

A Begg's funnel plot with pseudo

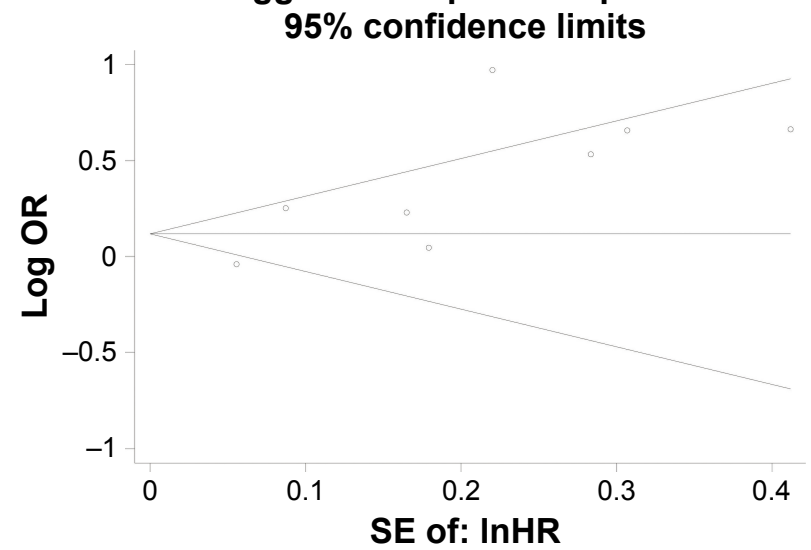

B Begg's funnel plot with pseudo $95 \%$ confidence limits

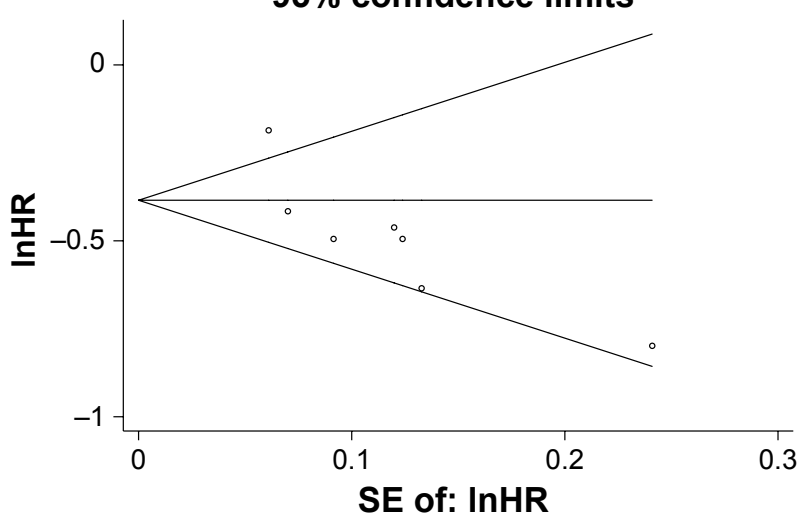

Figure S3 (Continued) 
C
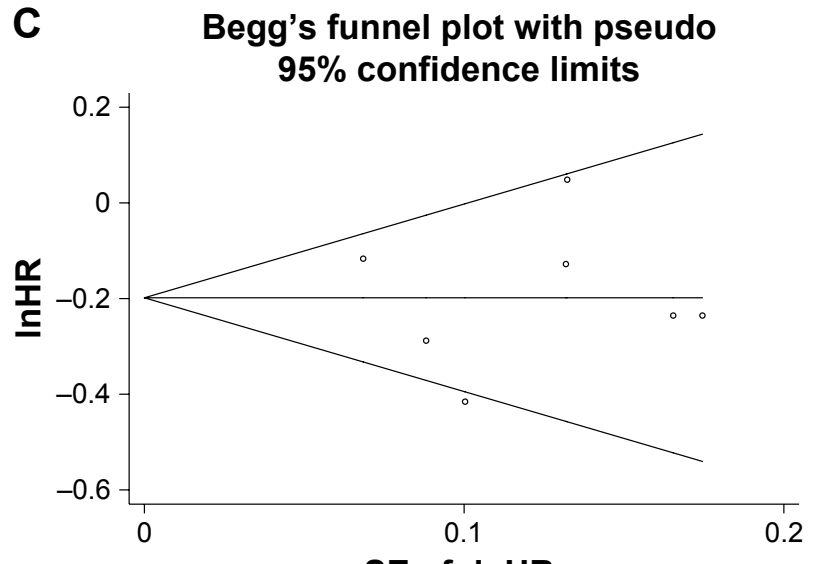

SE of: InHR

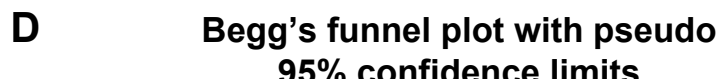

$95 \%$ confidence limits

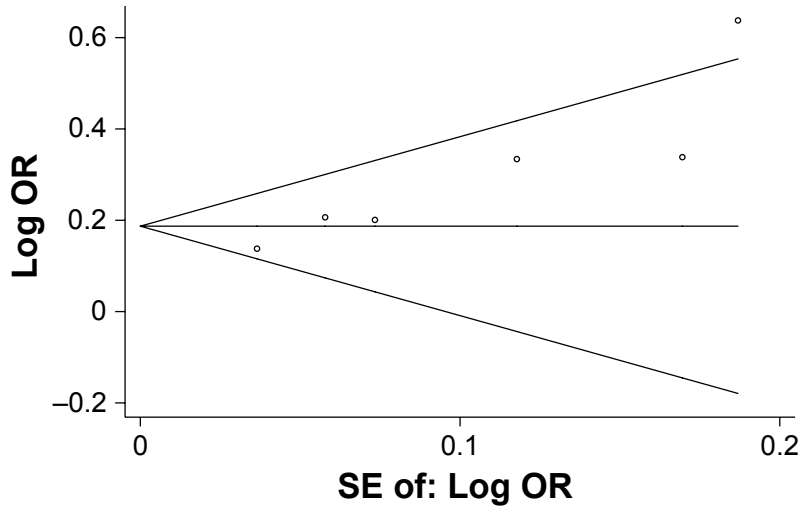

Figure S3 Sensitivity analysis. Bars $=95 \%$ confidence interval. Results symbolized by omitting each study in turn. (A) Overall response rate (ORR); (B) progression-free survival (PFS); (C) overall survival (OS); (D) grade 3/4 treatment-related adverse events.

A Egger's publication bias plot

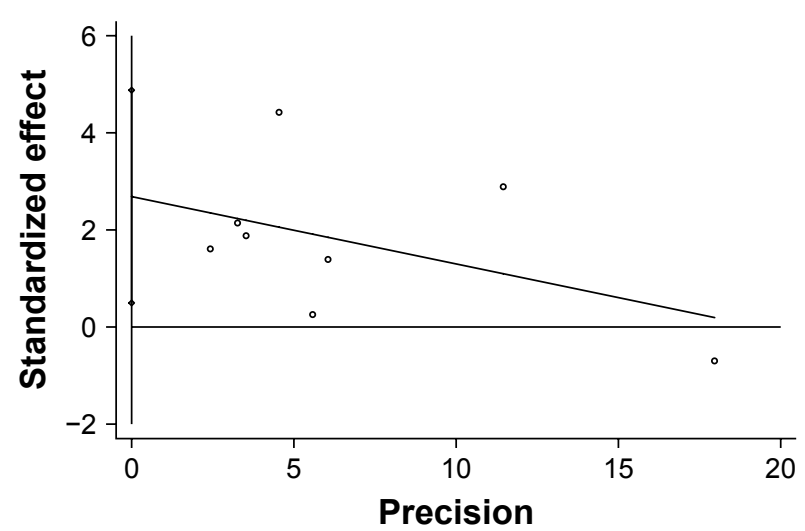

C

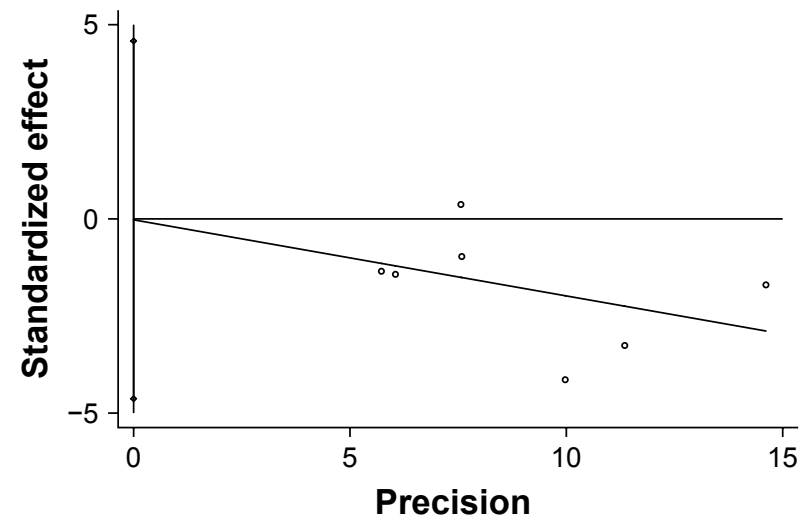

B Egger's publication bias plot

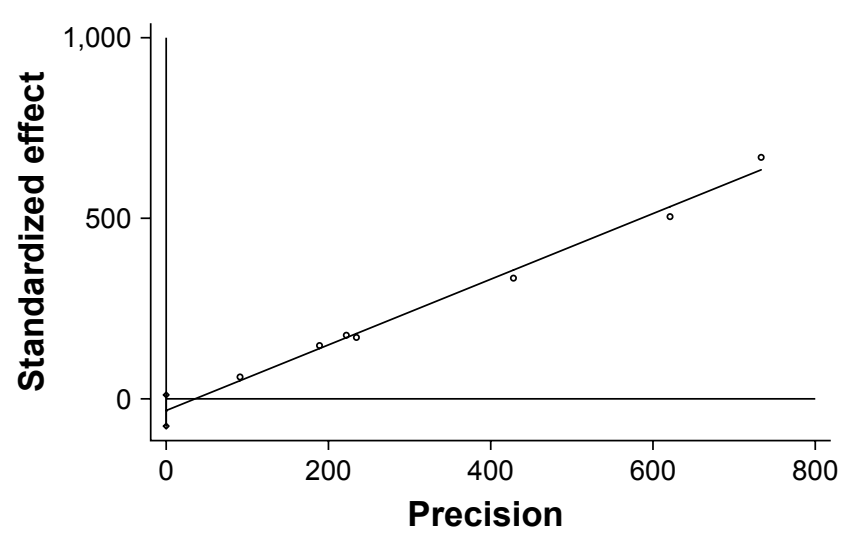

D Egger's publication bias plot

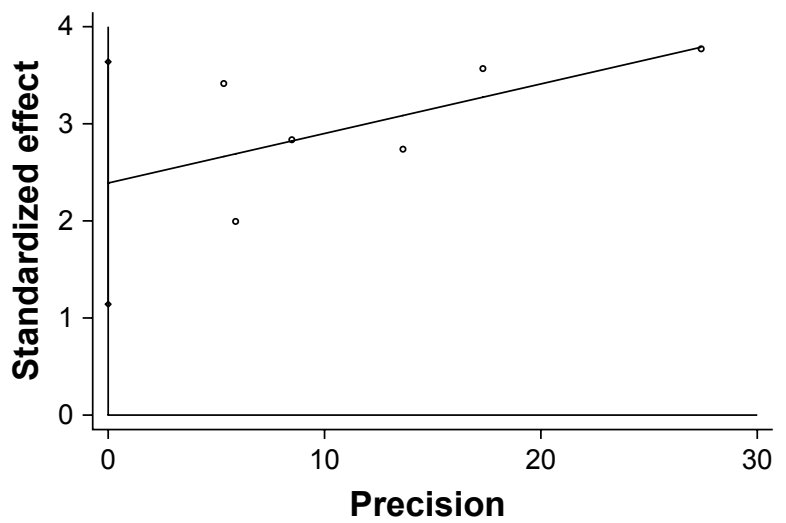

Figure S4 Publication bias assessed by Egger's test. (A) Overall response rate (ORR); (B) progression-free survival (PFS); (C) overall survival (OS); (D) grade 3/4 treatmentrelated adverse events. 


\section{A}

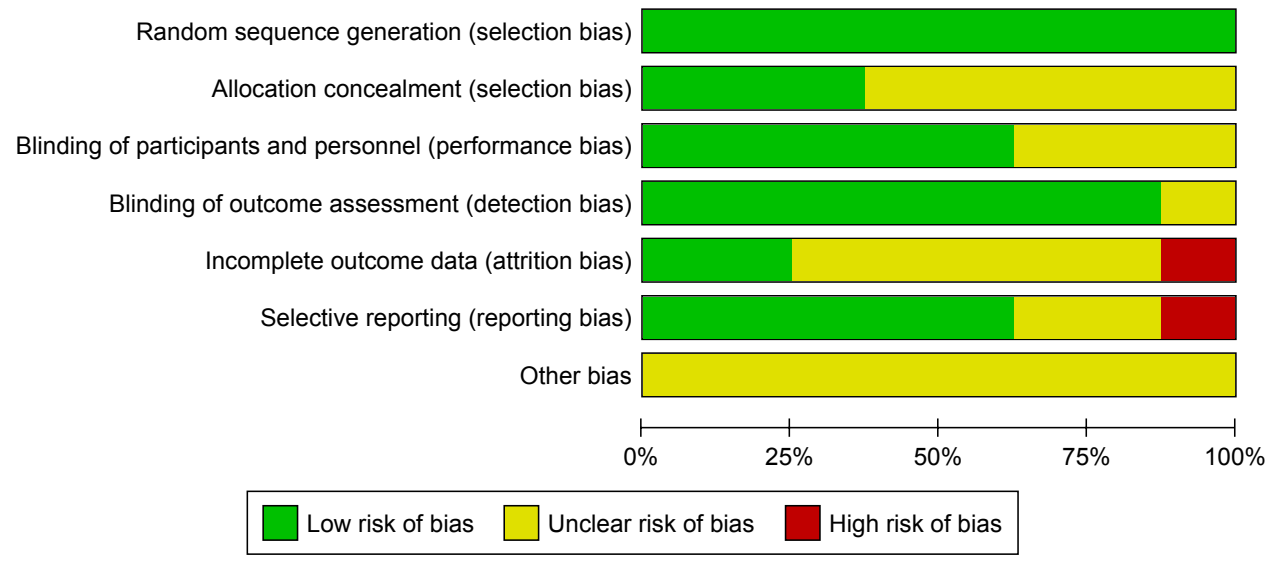

B

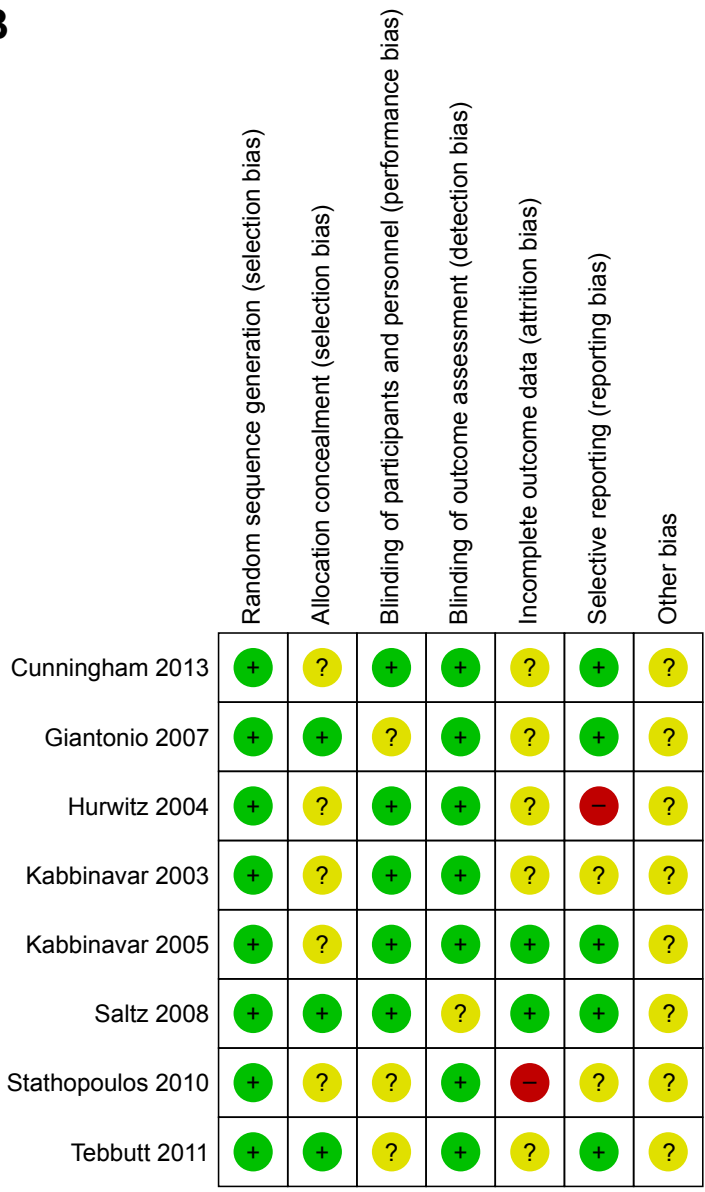

Figure S5 Risk of bias assessment; (A) Risk of bias graph: review authors' judgments about each risk of bias item presented as percentages across all included studies. (B) Risk of bias summary: review authors' judgments about each risk of bias item for each included study.

OncoTargets and Therapy

\section{Publish your work in this journal}

OncoTargets and Therapy is an international, peer-reviewed, open access journal focusing on the pathological basis of all cancers, potential targets for therapy and treatment protocols employed to improve the management of cancer patients. The journal also focuses on the impact of management programs and new therapeutic agents and protocols on

\section{Dovepress}

patient perspectives such as quality of life, adherence and satisfaction The manuscript management system is completely online and includes a very quick and fair peer-review system, which is all easy to use. Visit http://www.dovepress.com/testimonials.php to read real quotes from published authors.

Submit your manuscript here: http://www.dovepress.com/oncotargets-and-therapy-journal 